\title{
An Ontology-based Question Answering Method with the use of Query Template
}

\author{
Lakshmi Palaniappan, \\ Asso. Prof. Department of Information Technology \\ R.V.S. College of Engg. \& Tech., \\ Dindigul, India
}

\author{
Dr. N. Sambasiva Rao \\ Pro Vice chancellor, \\ Prist University, \\ Pondicherry, India
}

\begin{abstract}
This paper presents a new method for ontology-based question answering (QA) with the use of Query template for Dining Ontology as the domain (Service of the restaurant is called dining). Hypothesis questions and query templates can be produced from domain ontology. Pre-generation of hypothesis questions on the basis of the domain ontology and textual entailment. Semantic web is the expression of meaning of the content. Semantic web based gives instant access. Reusability is enhanced. This method is not domain specific. It focuses on the RDF, Ontology model and Web ontology language. Design of the ontology created can be viewed. Query processing is faster using text pool, dining ontology is developed.
\end{abstract}

\section{Keywords:}

Ontology, RDF, Semantic web, query template.

\section{INTRODUCTION}

Since modelling ontologies is a tedious and costly task, it is always important to demonstrate the advantages by applying ontologies in Software Engineering.

So the logic-based formalisms in the context of the semantic web effort is an important factor. Activities by the W3C and others have helped by the standards like RDF or OWL.

Another important factor is the flexibility of ontologies,. ontologies are well-suited to combine information from various sources and infer new facts based on this. Also, the flexibility allows to extend existing ontologies very easy, thus fostering the reuse of existing work.

In contrast to traditional knowledge-based approaches, e.g. formal specification languages, ontologies seem to be well suited for an evolutionary approach to the specification of requirements and domain knowledge.

Ontology is the specification of a concepts. Conceptualization is a simplified view that represent the purposes. Every ontology includes a dictionary with explanation of the terms and indications and shows relations. The ontology represents conceptual description of the specific content, to identify appropriate terms and relationship in a given knowledge domain. Ontologies show a hierarchical dependents of the terms together with descriptions, explanations and definitions. New user able to understand its use and incorporate the concepts in a knowledge domain. Ontology gives graphical representation by ontoviz and owlviz.
Software modelling languages and methodologies can benefit from the integration with ontology languages such as RDF and OWL in various ways, e.g. by reducing language ambiguity, enabling validation and automated consistency checking. Here, ontologies provide a unified representation for both problem domain and source code, thus enabling easier cross-references among both information spheres. Moreover, it is easy to create arbitrary views on the source code (e.g. concerning a variable). Reasoning is applied to create those views, e.g. to find all places where a variable is accessed either directly or indirectly. Ontologies provide a mechanism to capture knowledge about the problem domain.

Using RDF ontology, every provider is free to add or subclass concepts from the initial version without being at risk to become incompatible. The ontology document is present in RDF languages. Query templates used for data extraction. RDF is an abstract model with several serialization format, so particularly resource are triple is encoded varies from format to format in [2]. The RDF data model is based on a class diagram, and it make statements, about resources in the form of subject-predicate-object expressions. This expressions are known as triples. The subject denotes the resource, the predicate denotes aspects of the resource and expressed a relationship between the subject and the object.

This project deals with creation of ontology for restaurant operation, that is service of the restaurant may be called as dining, hence dining ontology created.

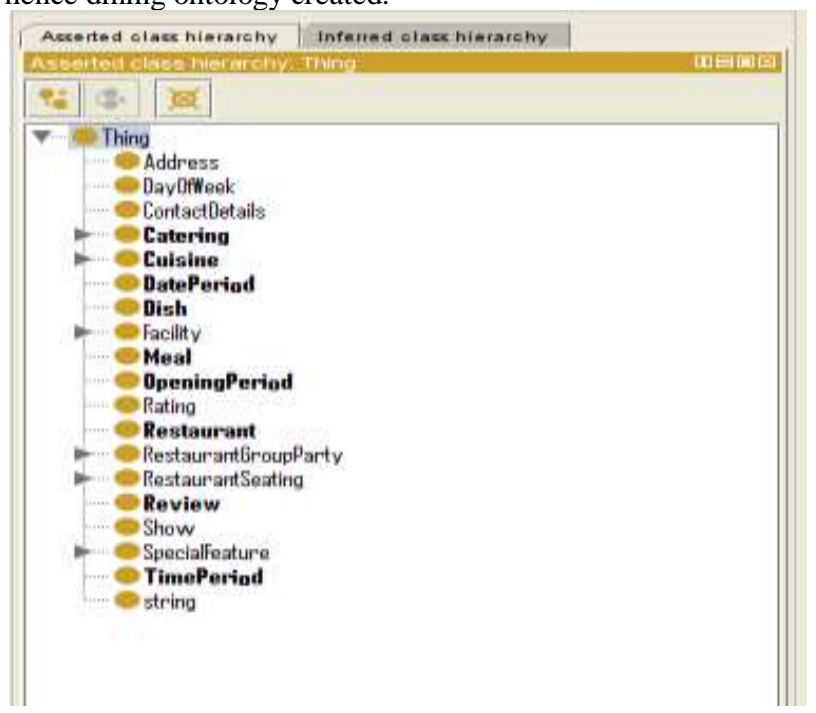

An ontology is a formal representation of the knowledge by a set of concepts within a domain and the relationships between those concepts. An ontology consists of individuals, classes, properties. 
Individuals represent objects in the domain. The project deals with creation of ontology for dining .It is one of the service in restaurant, in dining ontology properties are binary relation on individuals. In this domain, dining ontology properties such as is a relationship is created. Various properties available are transitive, functional, inverse, and symmetric. Classes are interpreted as sets that contain individuals. Ontology is created using protege 4.0.2.The owlviz tab in protégé is used to view the design of ontology that is created. Apart from dlquery, query template plug in (add-in) created, to retrieve and store, hence reusability is enhanced.

Section 2 Reviews some related question answering systems. (Related work).

Section 3 Description of the domain ontology.

Section 4 Architecture of the dining ontology.

Section 5 Implementation

- Design of the dining ontology.

- Question answering using dlquery and query template. Section 6 Conclusion.

\section{RELATED WORK}

The availability of domain ontologies makes possible to semantically annotate questions and transform them into an ontological representation.

In question answering the issue of representing question as a structured format for data retrieval is not a trivial task. automatic question pattern generation for ontology based question answering was developed using sparql in 2008 referred in [5] work in this area can be traced back to the early databased QA systems. It involves a type of tourism site,Cinema, and a type of tourism event,MovieShow.

The availability of domain ontologies makes possible to semantically annotate questions and transform them into an ontological representation, an early ontology based question answer in textual entailment using sparql in [1]. Now the proposed work based on the future enhancement of textual entailment, that is by using query template QA retrieval. This web service poses a structured query expressed in OWL DL uses reasoned to check whether the content matches the query.

Answering question pattern generation for ontology-based question answering sparql used (2008) these "different" questions share the same query for data retrieval and obtain the same answers, and thus it is not worth processing each expression to produce the same query. The example may be what is the name of the movie which has the genre value. To address this problem, textual entailment was proposed as a solution to determine whether different expressions entail the same meaning and thus can be the same retrieval procedure.

An ontologies-based question answering method with the use of textual entailment using sparql (2009).In this tourism taken as domain for example the question may be what is the name of the hotel which has a star rating? This method is very appropriate for ontology based QA in restricted domain because hypothesis questions and query templates can be produced from a domain ontology. It also reveals a weakness that the produced hypothesis questions cannot cover all kinds of user questions. This can become particularly problematic for a large domain in this ontology. So to overcome the problem, in this paper we have proposed query template plug -in that is used in this paper using protégé 4.0.2.

In this paper protege 4.0.2 used hence apart from dlquery, query template as a tab created. as plug-in in protege. questions templates stored and hence reusability exists,. and owlviz tab in protege is viewed the design of ontology created.

\section{DESCRIPTION OF THE DOMAIN ONTOLOGY}

A representative part of the ontology is the service of the Restaurant as DINING ontology. It involves address, contact detail of the restaurant and about catering details, that is beverages available with cost to the customers, beer, wine with price. Whether home delivery available, self service needed. This ontology give details about cuisine, whether it is coffee shop cuisine, wine bar cuisine, icecream parlor cuisine, Indian cuisine, vegetarian cuisine and also about the Atmosphere, whether it is business \&casual atmosphere, dance floor available. dish details can be viewed and facility available as business dining ,children \&baby facility available, baby chair, baby laundry, children meal, children game area.

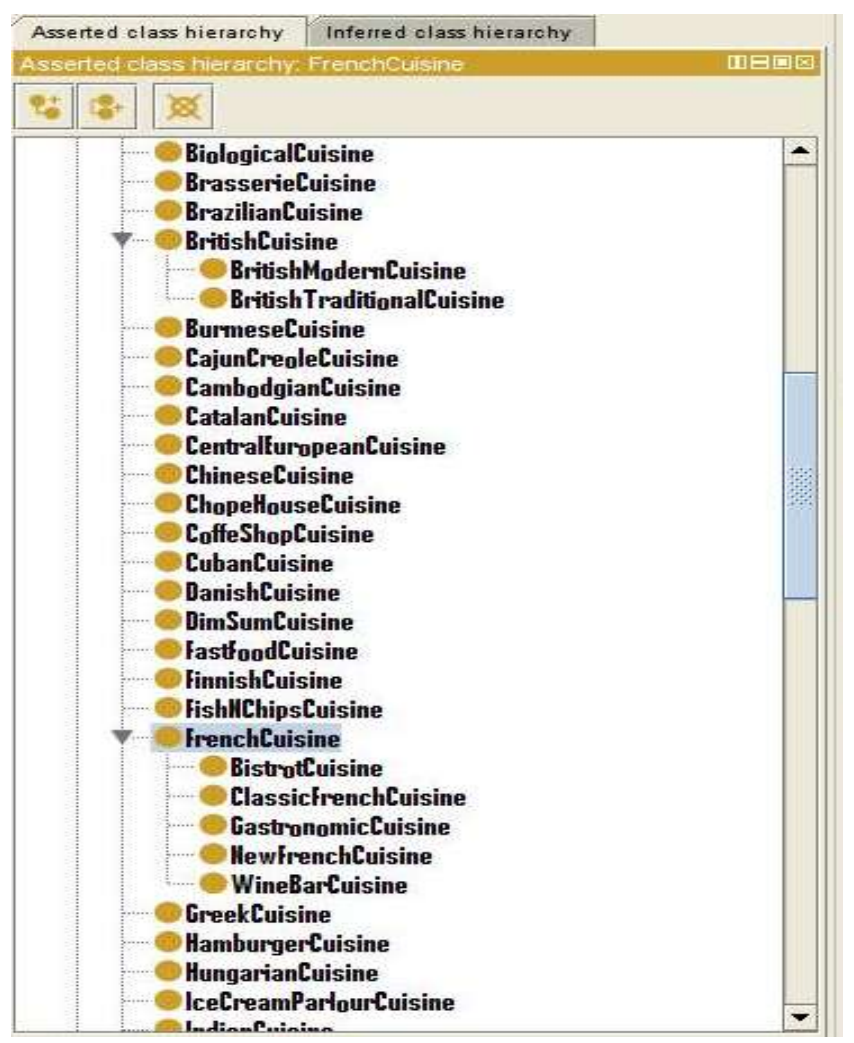



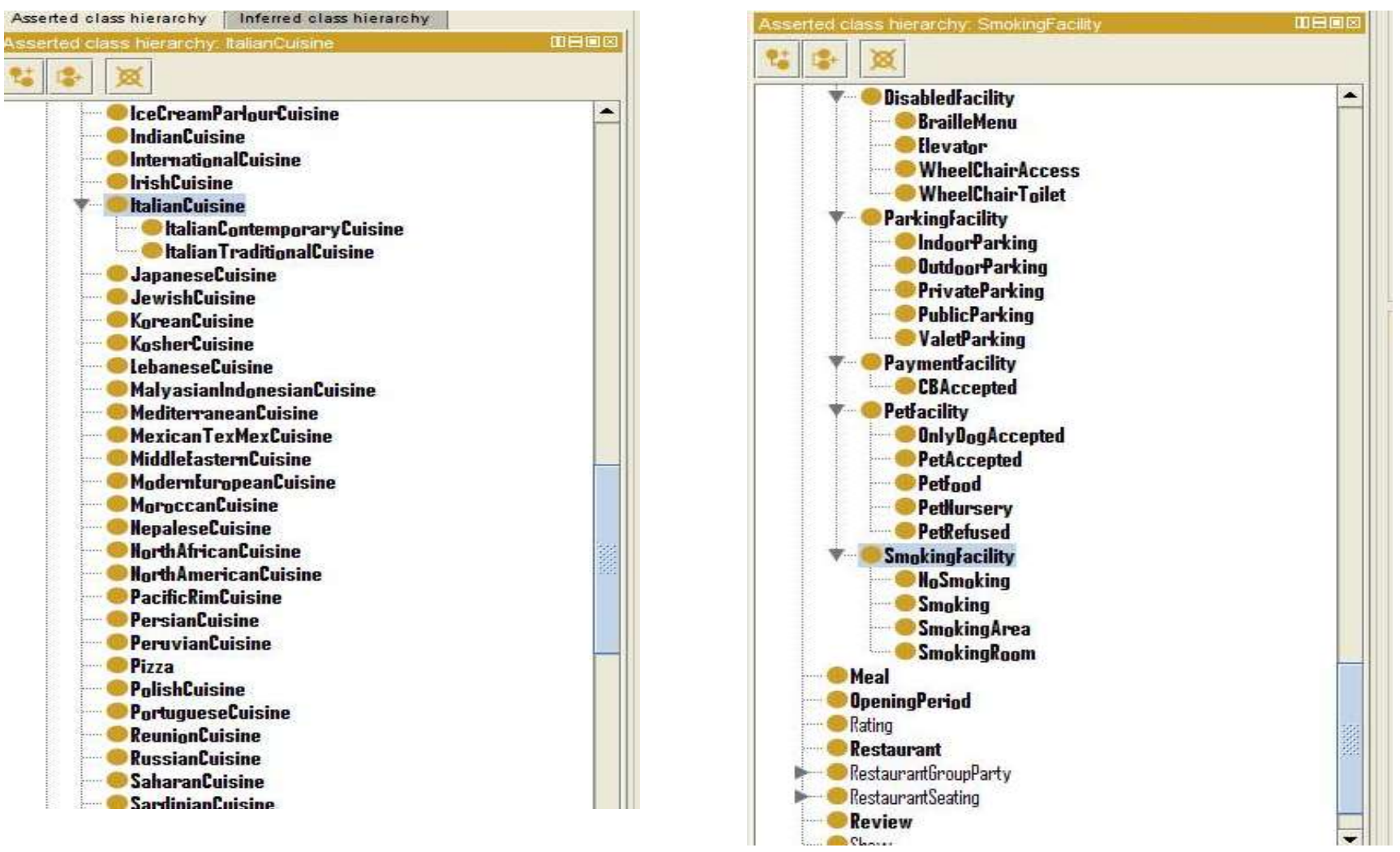

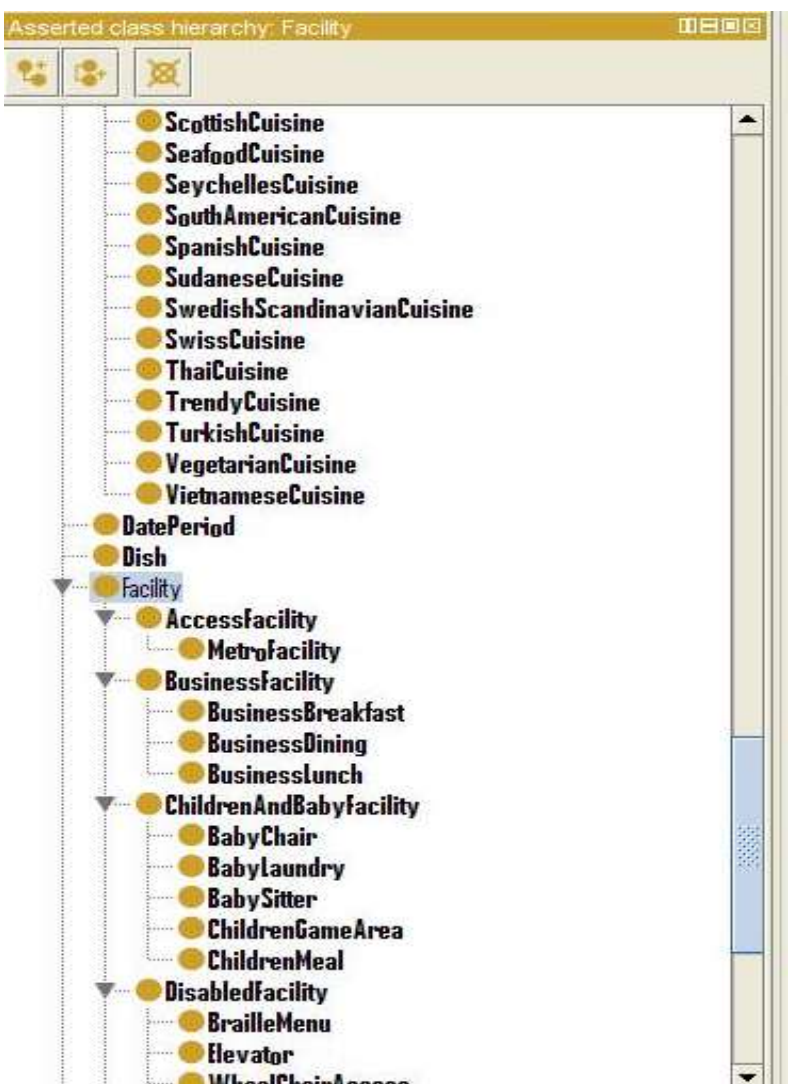

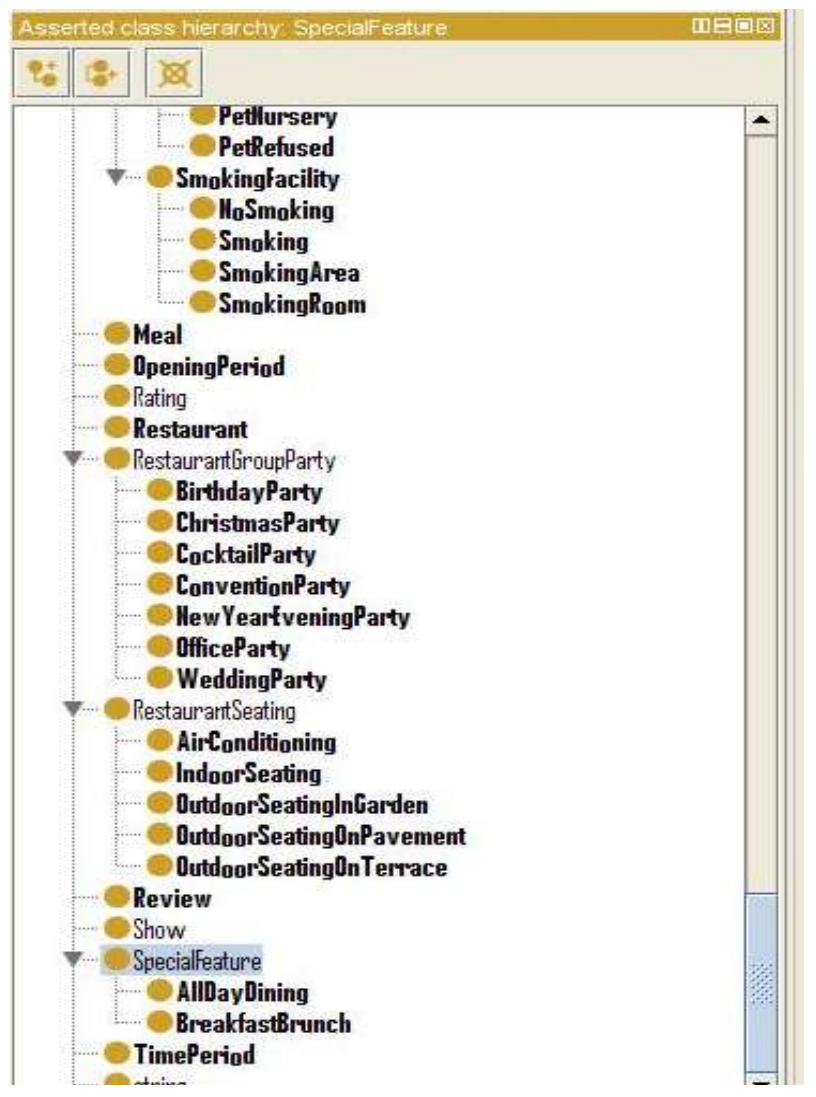


This ontology shows disabled facility, elevator exist, wheel chair access, wheel chair toilet exist \&also parking facility can be viewed as indoor parking, outdoor parking, private parking, public parking, payment facility is specified, wheather card accepted. whether pet facility exists, petfood, petnursery, smoking facility available, smoking room exists, smoking area exists, rating and restaurant group party \&restaurant seating. In restaurant group party, birthday party can be arranged, Christmas party, wedding party, new year party. In restaurant seating, air conditioning is available, indoor seating, outdoor seating \&also some special features are all day dining, breakfast brunch, time period and all these details can be viewed.

\section{ARCHITECTURE FOR DINING ONTOLOGY:}

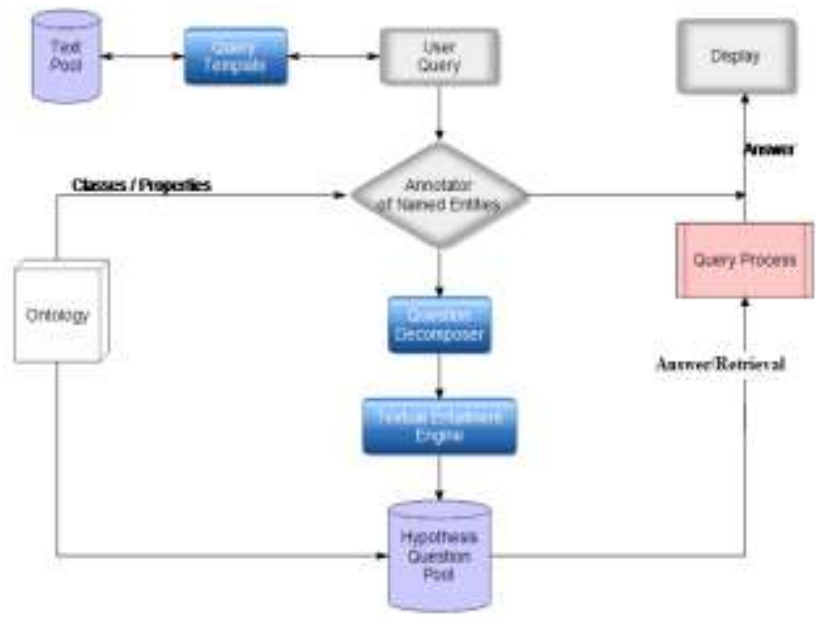

Figure shows the architecture of dining ontology as domain. User query will pass through annotator of named entities, then queries will be checked whether it is synchronized, after test by reasoner, the answer will be searched in the text pool and it is retrieved. Instead of going to database query template process queries faster. Thus reusability is enhanced.

\section{IMPLEMENTATION \\ 5.1 Design of the Dining Ontology}

The ontology designed in our project aims at providing a conceptualized description of the dining of the restaurant as domain. The ontology was encoded using the OWL DL language. It covers dining that in restaurant as about details of cuisine, facility, meal, rating restaurant seating and the relation ship between them.

From the point of view of design, the top-level classes fall into Categories. Main classes refers to the most important concepts in dining domain example: cuisine, facility, catering. Element classes refers to the elements of the main classes facility includes smoking Facility, disabled facility, payment facility, restaurant seating, airconditioning, indoor seating, outdoor seating. Attribute classes includes property of entity that is group of attributes of the main classes or element classes example price, size. These occurs in object prpperty and data property in our project using protege4.0.2.

To construct a knowledge base for providing potential answers to user questions, we annotated the original dining data obtained from the web using semantic markup derived from the designed OWL ontology. The dining data were encoded in the RDF format, which can be used to instantiate the ontology. To access RDF based knowledge query languages instead of sparql, protégé need to be used to retrieve specific contents from it for QA.

Ontoviz \&owlviz gives the graphical representation in owl.hence protégé owl is used to view the design. hence ontology and its properties created by using the owlviz we can get graphical representation.ontoviz is ontology visualization, hence meaningful relationship is created as Air conditioning is a restaurant seating.owlviz will show the subclass of the main class, hence in protégé 4.0.2 OWLVIZ is created shows both these semantic relationship.

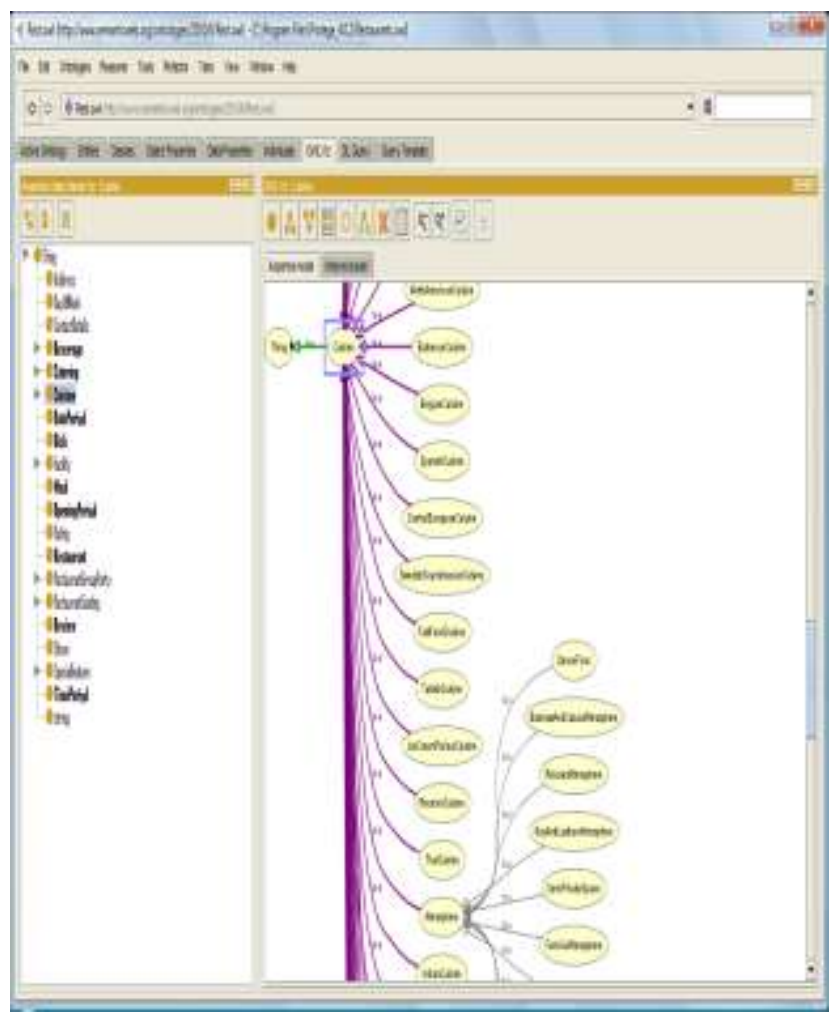

The owl viz tab in protégé is used to view the design of ontology created.

\subsection{Question Answering using dlquery and query template.}

In this section we focus on how to create question and answer retrieval using DLQUERY. By creating object property and data property, data are mapped. 

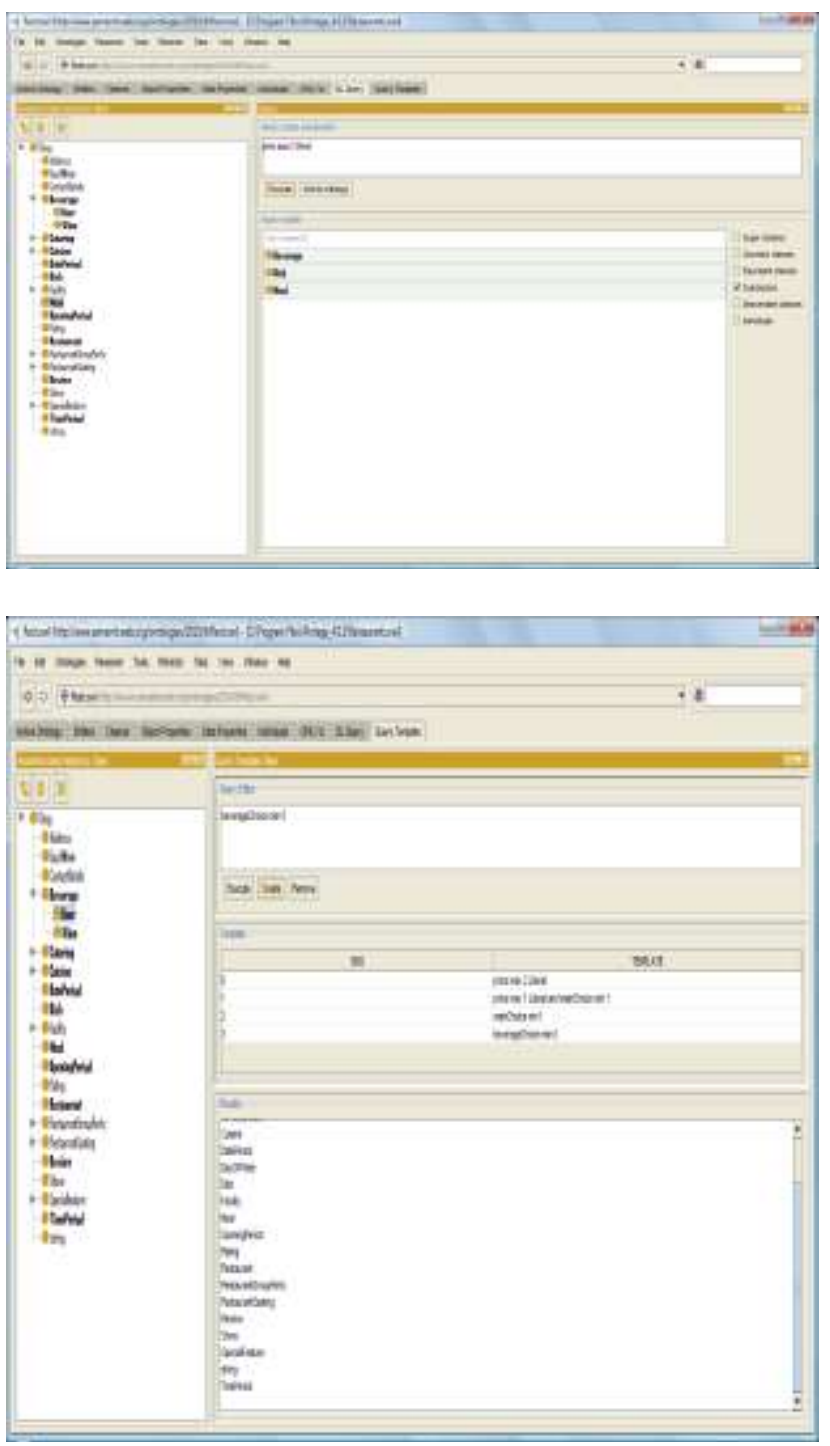

We can form questions and in the dlquery the question should be in the same way, otherwise it indicates error, after creating query answer will be retrieved. To parse the OWL ontology and derived all the properties associated with it, the Query Template plug-in created in java. These associated properties represent all possible items which can be queried for the instances in the class.

By using query template in protégé faster retrieval of data then sparql, and also the queries can be accessed from templates. REUSABILITY occurs by using this query template.

\section{CONCLUSION}

This paper presents an approach for dining ontology by question answering method using query template. It involves RDF, ontology model and web ontology language. This project is not domain specific already for question answering by textual entailment using sparql made, but they proposed query template may be used, hence protégé is used and query template plug in created and hence of reusability occurs with less time,hence apart from owlviz question checked by reasoner, if it is synchronized then answers can be retrieved. queries stored it forms templates, hence reusability exists. Like tourism as a domain, service of the restaurant details can be viewed easily. By query template data can be extracted quickly, for future enhancement this details can be viewed as IMAGE.

\section{REFERENCES}

1. Shiyan Ou, Dalila Mekhaldi, Constantin Orasan. (2009). An ontology-based question answering method with the use of textual entailment. In Proceedings of International Conference on Natural Language Processing and Knowledge Engineering 2009, pp. 212-219. Dalian, China, 24-27 September 2009. Publisher: IEEE

2. Frank Manola, Ericmiller, Resource Description Frame Work (RDF): Concepts and Abstract syntax, February 2004. http://www.w3.org/TR/2004/RECrdf-primer.

3. Basili, R.,Hansen, D.H.,Paggio, P.,Pazienza M.T., and Zanzotto F.M.2004. Ontological Resources and Question Answering. In Proceeding of the Workshop on Pragmatics of Question Answering held in conjunction with HLT-NAACL 2004. Morristown, NJ: ACL.

4. Applications of Ontologies in Software Engineering by Hans-Jorg Happel and Stefan Seedort.. FZI Forschungszentrum informatik, Forschungsgruppe information process engineering (IPE), (2008) Germany.

5. Shiyan Ou, Dalila Mekhaldi, Laura Hasler. Automatic Question Pattern Generation for Ontology-based Question Answering. Research Group in computational Linguistics University of Wolverhampton, UK. Proceeding of the Twenty-First International FLAIRS Conference (2008).

6. Vikash Kumar (2009) Master thesis Free University of Bolzano: An Ontology-based Approach for Interactive Question Answering: Experiments with the QALL-ME System. Advisor: Bernardo Magnini.

7. Negri M., Kouylekov M., Magnini B., Mehdad Y., and Elena Cabrio Towards Extensible Textual Entailment Engines: the EDITS Package. To appear in Proceedings of AI*IA 2009, Reggio Emilia, Italy, December 9-12, 2009.

8. Magnini B., Speranza M., and Kumar V. Towards Interactive Question Answering: An Ontology-Based Approach. In proc. of the Workshop on Semantic Computing and Multimedia Systems (SCMS 2009) held at the third IEEE International Conference on Semantic Computing (ICSC 2009), Berkeley, California, September 2009.

9. Negri M. and Kouylekov M. Question Answering over Structured Data: an Entailment-Based Approach to Question Analysis. In Proceedings of RANLP 2009, September 14-16, 2009, Borovets, Bulgaria.

10. Sacaleanu B., Spurk C., Orasan C., Ferrandez O., Kouylekov M., Negri M., and Ou S. Entailment-based Question Answering for Structured Data. In Proceedings of COLING 2008 (Demo Session), Manchester, UK, August 18-22, 2008. 